\title{
Cardiac Stem Cells Suppressed Cardiac Nerve Sprouting via Attenuated Oxidative Stress in a Rat Model of Myocardial Infarction
}

\author{
Jian Chen ${ }^{1, *}$, Shaoxin Zheng ${ }^{2}$, Huibao Long ${ }^{3}$, Tong Wang ${ }^{3}$ \\ ${ }^{1}$ Department of Cardiology, the Fifth Affiliated Hospital of Sun Yat-sen University, China \\ ${ }^{2}$ Department of Cardiology, the Sun Yat-sen Memorial Hospital of Sun Yat-sen University, China \\ ${ }^{3}$ Department of Emergency, the Sun Yat-sen Memorial Hospital of Sun Yat-sen University, China
}

Copyright $\bigcirc 2016$ by authors, all rights reserved. Authors agree that this article remains permanently open access under the terms of the Creative Commons Attribution License 4.0 international License

\begin{abstract}
Objective: To investigate whether cardiac stem cells (CSCs) can attenuate oxidative stress and improve sympathetic remodeling in rats following myocardial infarction (MI). Methods: Sprague-Dawley rats were randomly divided into three groups: 1) MI with CSCs transplantation (CSCs group, $\mathrm{n}=15$ ); 2) MI control (Control group, $\mathrm{n}=15$ ); 3) sham control group (Sham group, $\mathrm{n}=10$ ). Two weeks after surgery, the animals were subjected to intramyocardial injection. Four weeks after injection, left ventricular function, level of oxidative stress, cardiac nerve sprouting and nerve growth factor (NGF) expression were determined. Results: At 4 weeks after intramyocardial injection, fractional shortening (FS) and left ventricular ejection fraction (LVEF) markedly decreased in CSCs and control groups. However, systolic function were partly preserved in CSCs group compared with control group $(P<0.01)$. The myocardial malondialdehyde (MDA) content, MDA/Superoxide Dismutase (SOD) ratio, NGF expression and density of tyrosine hydroxylase (TH)-positive nerve in peri-infarct zone was significantly increased in the control group and CSCs group than the sham group (all $P<0.01$ ). However, all of these valuables were decreased in CSCs group when compared with control group (all $P<0.01$ except for NGF expression $P<0.05$ ). Furthermore, a positive correlation was observed between the degree of sympathetic remodeling and the level of MDA/SOD ratio $\left(r=0.86, \quad \mathrm{R}^{2}=0.75\right)$. Conclusion: CSCs suppressed sympathetic remodeling after MI probably via attenuated oxidative stress and downregulated NGF expression, which in turn leads to a promising treatment for SCD post MI.
\end{abstract}

Keywords Cardiac Stem Cells, Myocardial Infarction, Sympathetic Remodeling, Oxidative Stress, Nerve Growth Factor, Sudden Cardiac Death

\section{Introduction}

Sudden cardiac death (SCD) is one of the most common and often the first manifestation of coronary heart disease and account for $50 \%$ of coronary heart disease deaths and $15 \%$ to $20 \%$ of all deaths in developed countries [1]. Life-threatening ventricular arrhythmias occur often in chronic myocardial infarction (MI) but the mechanism remains unclear. Recently, studies have shown that sympathetic remodeling post MI plays an important role in life-threatening ventricular arrhythmias and even SCD [2-4]. Increasing evidences show oxidative stress may contribute to sympathetic remodeling via increasing local and circulatory nerve growth factor (NGF) [5-8].

Laboratory experiments and recent clinical trials suggest that cell-based therapies for MI can improve cardiac function. Resident cardiac stem cells (CSCs) appears as a promising stem cell because of the ability of differentiating into cardiomyocytes, endothelial cells, vascular smooth muscle cells, and fibroblasts $[9,10]$, thus, demonstrating improvement of left ventricular function. Furthermore, our previous study has shown that rats post MI treated with CSCs improved cardiac electrophysiological stability and ventricular fibrillation threshold [11].

Some types of stem cells demonstrate significant capability to mitigate oxidative stress and inflammatory reaction during treating diabetic cardiomyopathy or renal disease [12]. Given that improved sympathetic remodeling is associated with fewer incidences of arrhythmias, therefore, we hypothesized that CSCs can attenuate sympathetic remodeling process through suppression of oxidative stress accompanied by reduction of NGF, which, in turn, probably result in a decrease in the incidence of arrhythmias.

\section{Materials and Methods}




\subsection{Isolation and Culture of Cardiac Stem Cells}

CSCs were isolated, characterized, and cultured as described previously by our group [11]. The 3-day-old Sprague-Dawley (SD) rats were dipped into $75 \%$ ethanol for 30 seconds for sterilization after anaesthetization. The hearts were excised and minced into 1-mm3 pieces. After wash with phosphate buffer solution (PBS), $2-3 \mathrm{ml}$ of $0.2 \%$ trypsin was added for $5 \mathrm{~min}$ of digestion, followed by $2-3 \mathrm{ml}$ of $0.1 \%$ collagenase II for $5 \mathrm{~min}$ for additional digestion, and subsequent elimination of the digestive liquid. The procedure was then repeated for an additional three times. The pieces were then washed twice with complete explant medium (supplemented with Iscove's modified Dulbecco's medium, $10 \%$ fetal calf serum, 100 units $/ \mathrm{ml}$ penicillin $\mathrm{G}, 100$ $\mu \mathrm{g} / \mathrm{ml}$ streptomycin, $2 \mathrm{mmol} / \mathrm{L}$ L-glutamine, and $0.1 \mathrm{mmol} / \mathrm{L}$ 2-mercaptoethanol), then transferred into a $25-\mathrm{cm} 2$ flask, and cultured with $1 \mathrm{ml}$ of complete explant medium in an atmosphere of $5 \%$ carbon dioxide at $37^{\circ} \mathrm{C}$ for 12 hours. An additional complete explant medium $(3-4 \mathrm{ml})$ was then added. At $90 \%$ confluence, the cells were trypsinized $(0.25 \%$ Trypsin/2.21 mM ethylenediaminetetraacetic acid) and were passed into $25-\mathrm{cm} 2$ flasks at $1: 2$ ratios. The cells were expanded to third-passage prior to injection into the animals. Cultured CSCs were analyzed by fluorescence-activated cell sorting (FACScan flow cytometer, Becton Dickinson, Sparks, MD) as reported previously[11,13].

\subsection{Animals and Experimental Designs}

Male SD rats, weighing between $300 \mathrm{~g}$ and $350 \mathrm{~g}$, age between 8 weeks and 9 weeks (Experimental Animal Center of Sun Yat-sen University, Guangzhou, PR China) were housed in a standard animal facility with 12-hour on/off light conditions. The animal research study protocol was in compliance with 'The Guide for the Care of Use of Laboratory Animals' published by the National Institute of Health (NIH Publication No. 85-23, revised 1996) and approved by approved by the institutional animal care committee.

The rats were randomly assigned to the following experimental groups: 1) MI with CSCs transplantation (CSCs group, $\mathrm{n}=15$ ), rats intramyocardial injected with $5 \times 106 / 0.1 \mathrm{ml}$ of CSCs in PBS; 2) MI control (Control group, $\mathrm{n}=15$ ), rats intramyocardial injected $0.1 \mathrm{ml}$ of PBS; 3 ) sham control group (Sham group, $\mathrm{n}=10$ ), rats underwent sham operation without suture tie-down and were intramyocardial injected $0.1 \mathrm{ml}$ of PBS.

\subsection{Preparation for Rat Myocardial Infarction Model and Intramyocardial Injection}

The animal MI-model has been described previously by our group [11, 14]. Briefly, SD rats were fasted overnight except for free access to water. The animals were anesthetized by intraperitoneal injection of pentobarbital (45 $\mathrm{mg} / \mathrm{kg}$ ). The trachea was orally intubated and mechanically ventilated with room air. The electrocardiogram (lead II) was continuously monitored by MP 150 Biopac System (Santa Barbara, CA, USA). A thoracotomy was performed via the left fourth intercostal space. The pericardium was incised, and the left atrial appendage was elevated to expose the left anterior descending coronary artery. The left anterior descending coronary artery was ligated by using a 5/0 nylon suture. The chest was closed with a soft tube in the cavity to withdraw air or blood. Successful occlusion was confirmed electrocardiographically by ST segment elevation [13]. Animals were allowed to recover from anesthesia and were then returned into their cages for 2 weeks. Intramuscular injection of ketorolac $(0.4 \mathrm{mg} / \mathrm{kg})$ was administered post-operatively for analgesia.

Two weeks after the surgery, a new thoracotomy was performed as previously described. All survived rats received multiple intramyocardial injections at the peri-infarct zone with $1-\mathrm{ml}$ syringe. Successful injection was ascertained by the formation of a bleb covering peri-infarct zone.

\subsection{Echocardiographic Measurements}

Left ventricular systolic function was studied by transthoracic echocardiography before MI induction, 2 weeks and 6 weeks after MI induction. Animals were scanned by transthoracic echocardiography (GE Vivid 7) with a $12 \mathrm{~S}$ pediatric probe. Left ventricular end diastolic dimension, left ventricular end systolic dimension and heart rate were measured and recorded. Indices of left ventricular systolic function, fractional shortening (FS) and left ventricular ejection fraction (LVEF) were calculated. All measurements were averaged on three consecutive cardiac cycles and were analyzed by two independent observers who were blinded to the treatment status of the animals.

\subsection{Tissue Collection}

Four weeks after CSCs or PBS injection, the heart was harvested under pentobarbital anesthesia $(100 \mathrm{mg} / \mathrm{kg})$ by intraperitoneal injection, and the left ventricular was then cut into three through the infarcted myocardium. One was immediately stored in $-80{ }^{\circ} \mathrm{C}$ for Western-blot, the other one was fixed in $4 \%$ formalin for immunohistochemistry, and the rest was for determination of oxidative stress biomarkers. The infarct zone was visually identified by a mottled and pale discoloration; the myocardium extending $0.5-1.0 \mathrm{~mm}$ from the infarct was defined as the peri-infarct zone. To avoid mixing the non-infarcted left ventricular free wall with the peri-infarct zone, myocardial areas extending 1-2 $\mathrm{mm}$ from the peri-infarct zone were not included[14,15].

\subsection{Determination of Superoxide Dismutase (SOD) Activity and Malondialdehyde (MDA) Level}


Tissue samples obtained from the peri-infarct zone of left ventricle was homogenized in $1.0 \mathrm{ml}$ of $20 \mathrm{mmol} / \mathrm{L}$ Tris $-\mathrm{HCl}$ $(\mathrm{pH}$ 7.4) in ice-cold condition, containing $5 \mathrm{mmol} / \mathrm{L}$ butylated hydroxytoluene. SOD activity and MDA level were determined by using commercially available kits (Jiancheng Bioengineering Institute, Nanjing, China) according to the manufacturer's introduction. The protein concentration was determined with the BCA protein assay reagent kit (Pierce, USA).

\subsection{Immunohistochemistry}

Samples taken from the peri-infarct zone were routinely processed and three sections of each sample were used for immunohistochemical study. Tyrosine hydroxylase (TH) is a marker of sympathetic nerves was examined. The primary antibodies used in this study were anti-TH (1:500, Abcam, USA). Nerve densities were determined by Image-Pro Plus 6.0 analysis system (Media Cybernetics, USA). The density of TH positive nerve was expressed as the nerve area divided by the total area examined $\left(\mu \mathrm{m}^{2} / \mathrm{mm}^{2}\right)$. Each slide was examined under a microscope and nerve-density was calculated by scoring 5 randomly-selected microscopic fields $[2,14]$.

\subsection{Western-blot}

Equal amounts of protein $(50 \mu \mathrm{g})$ were separated on 8-16\% Tris-glycine gel (Novex, Invitrogen, CA, USA) and transferred to a PVDF membrane. After blocking with 5\% skim milk, primary antibody against a goat polyclonal anti-NGF antibody (1:1000, Cambridge, UK) and a mouse monoclonal anti-GAPDH antibody (1:500, Beyotime, China) were incubated overnight at $4{ }^{\circ} \mathrm{C}$ followed by incubation with horseradish peroxidase-conjugated secondary antibody. Immunoreactivity was enhanced by chemiluminescence kit (Beyotime, China) and exposed to film. All the protein expressions were normalized to GAPDH protein content. The density of bands on Western-blot was quantified by using a Bio-Rad image system (Hercules, CA).

\subsection{Statistical Analysis}

Values were presented as means \pm SD. All data analysis was performed with the use of SPSS 22.0 statistical software (SPSS Inc., Chicago, IL, USA). One-way ANOVA followed by LSD correction for multiple comparisons was used for comparing the difference in all variables between groups. Pearson's correlation analysis was used to determine the correlation between the density of TH-positive nerve and MDA/SOD ratio. Statistical significance was defined as $P<0.05$.

\section{Results}

\subsection{Left Ventricular Systolic Function}

Two weeks after MI induction, FS and LVEF were markedly decreased when compared with baseline, which indicates the development of significant left ventricular dysfunction post MI; both of FS and LVEF were virtually indistinguishable between the control and CSCs groups, which demonstrates that the similar degree of left ventricular systolic functional deterioration at the time of treatment. As expected, in the control group, FS and LVEF represented downward trend but no significant change during the following 4 weeks $(P>0.05)$; in contrast, at 6 weeks, FS and LVEF of CSCs group were partly preserved when compared with 2 weeks $(P<0.01)$. As a consequence, at 6 weeks, FS and LVEF were higher in the CSCs group than in the control group $(P<0.01)$. Thus, transplantation of CSCs improved left ventricular systolic function. (Table 1)

Table 1. Echocardiographic assessment of left ventricular systolic function

\begin{tabular}{|c|c|c|c|c|}
\hline Characteristic & Sham & Control & $\mathrm{CSCs}$ & $p$ Value \\
\hline \multicolumn{5}{|l|}{ FS (\%) } \\
\hline $\mathrm{BL}(\mathrm{n})$ & $53.0 \pm 1.83(10)$ & $52.5 \pm 3.50(15)$ & $51.3 \pm 3.58(15)$ & 0.362 \\
\hline $2 \mathrm{~W}(\mathrm{n})$ & $51.5 \pm 3.67(9)$ & $22.4 \pm 3.32(11)^{1), \mathrm{a})}$ & $22.2 \pm 4.46(12)^{1), a)}$ & $<0.01$ \\
\hline 6W (n) & $51.5 \pm 3.91(9)$ & $21.2 \pm 3.47(8)^{1), a)}$ & $27.3 \pm 2.90(11)^{1), 2), a), b)}$ & $<0.01$ \\
\hline \multicolumn{5}{|l|}{ LVEF (\%) } \\
\hline $\mathrm{BL}(\mathrm{n})$ & $77.9 \pm 1.74(10)$ & $77.4 \pm 3.33(15)$ & $76.1 \pm 3.47(15)$ & 0.335 \\
\hline $2 \mathrm{~W}(\mathrm{n})$ & $76.4 \pm 3.65(9)$ & $39.6 \pm 5.16(11)^{1), a)}$ & $39.3 \pm 6.79(12)^{1), a)}$ & $<0.01$ \\
\hline 6W (n) & $76.4 \pm 3.92(9)$ & $37.8 \pm 5.46(8)^{1), a)}$ & $47.1 \pm 4.24(11)^{1), 2), a), b)}$ & $<0.01$ \\
\hline
\end{tabular}


All data are expressed as mean \pm SD. FS, left ventricular fractional shortening; LVEF, left ventricular ejection fraction; BL: baseline; $2 \mathrm{~W}$ : two weeks post $\mathrm{MI}$ induction; 6W: six weeks post MI induction (four weeks after intramyocardial injection). 1) $\mathrm{P}<0.01$, compared with Sham; 2) $\mathrm{P}<0.01$, compared with Control; a) $\mathrm{P}<0.01$, compared with $\mathrm{BL}$; b) $\mathrm{P}<0.01$, compared with $2 \mathrm{~W}$.

\subsection{Effect of Cardiac Stem Cells Transplantation on Levels of Oxidative Stress Biomarkers}

SOD activity was markedly decreased in control group $(59 \pm 14 \mathrm{U} / \mathrm{mg})$ compared with sham group $(132 \pm 16 \mathrm{U} / \mathrm{mg}$, $P<0.01)$. The CSCs transplantation resulted in higher SOD activity compared with control group $(102 \pm 10 \mathrm{U} / \mathrm{mg}$, $P<0.01$ vs. control group. Fig. 1A). In contrast, myocardial MDA content was prominently increased in control group $(3.22 \pm 0.17 \mathrm{nmol} / \mathrm{mg})$ compared with sham group $(1.44 \pm 0.15$ $\mathrm{nmol} / \mathrm{mg}, P<0.01)$ and significantly decreased in CSCs group $(2.50 \pm 0.30 \mathrm{nmol} / \mathrm{mg}, P<0.01$ vs. control group. Fig.
1B). The MDA/SOD ratio, an index of oxidative stress [16, 17], was significantly elevated in control group compared with sham group. While, it was reduced by CSCs transplantation compared with control group (both $P<0.01$. Fig. 1C).

\subsection{Effect of Cardiac Stem Cells Transplantation on Sympathetic Remodeling}

In contrast to very few TH-positive nerve in normal tissues of sham group (Fig. 2A), peri-infarcted ventricular tissues showed very high nerve density and actively arborizing large nerves (Fig. 2B-2C). The density of $\mathrm{TH}$ positive nerve in peri-infarct zone was significantly higher in the control group $\left(4151 \pm 780 \mathrm{\mu m}^{2} / \mathrm{mm}^{2}\right)$ and CSCs group $\left(2428 \pm 601 \mu \mathrm{m}^{2} / \mathrm{mm}^{2}\right)$ than the sham group $(950 \pm 470$ $\mu \mathrm{m}^{2} / \mathrm{mm}^{2}$, both $P<0.01$ ). However, a significant reduction in TH positive nerve densities was observed in CSCs group when compared with control group $(P<0.01$, Fig. 2D).
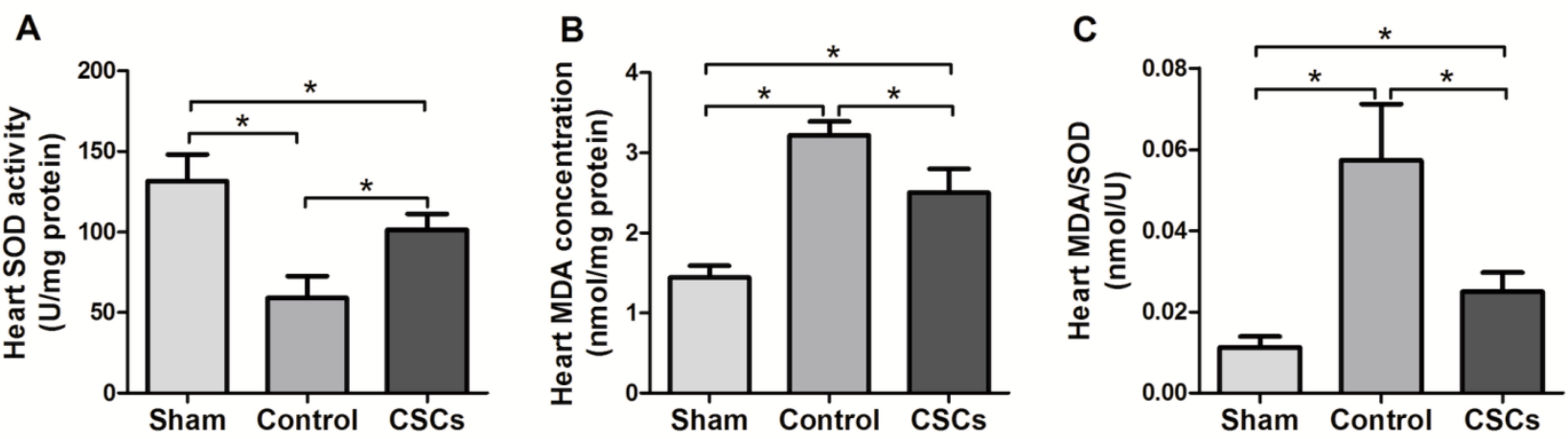

Figure 1. Bar graph showed SOD activity (A), MDA concentration (B) and the MDA/SOD ratio 4 weeks after intramyocardial injection. SOD: superoxide Dismutase, MDA: malondialdehyde. All data are expressed as mean $\pm \mathrm{SD}, *$ denotes $P<0.01$.
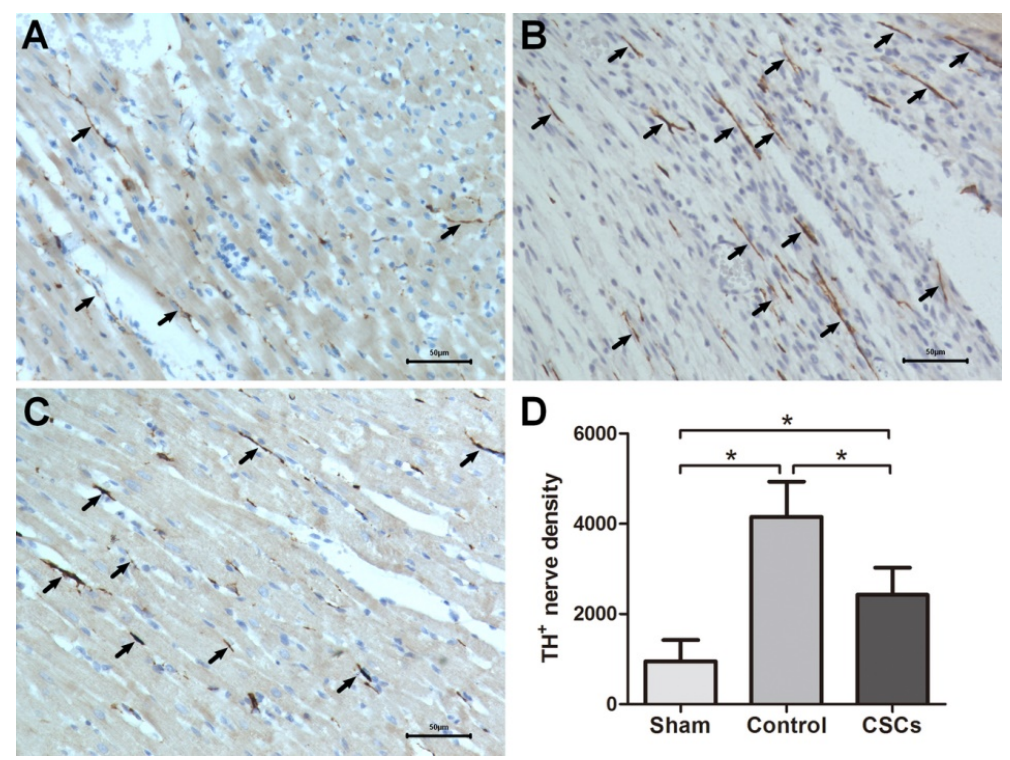

Figure 2. Examples of TH-positive nerve with anti-TH at peri-infarct zone (arrows). There were more TH-positive nerve in the control group (B) and CSCs group (C) than in the sham group (A). CSCs transplantation significantly decreased TH nerve density (D). Magnification $\times 400$, scale bar $=50 \mu \mathrm{m}$. All data are expressed as mean $\pm \mathrm{SD}, *$ denotes $P<0.01$ 


\subsection{Correlation of Sympathetic Remodeling with the Level of Oxidative Stress}

We further analyzed the correlation between sympathetic remodeling and the level of oxidative stress. A positive correlation was observed between TH positive nerve density and the MDA/SOD ratio in peri-infarct zone $(r=0.86$, $\mathrm{R}^{2}=0.75$. Fig.3).

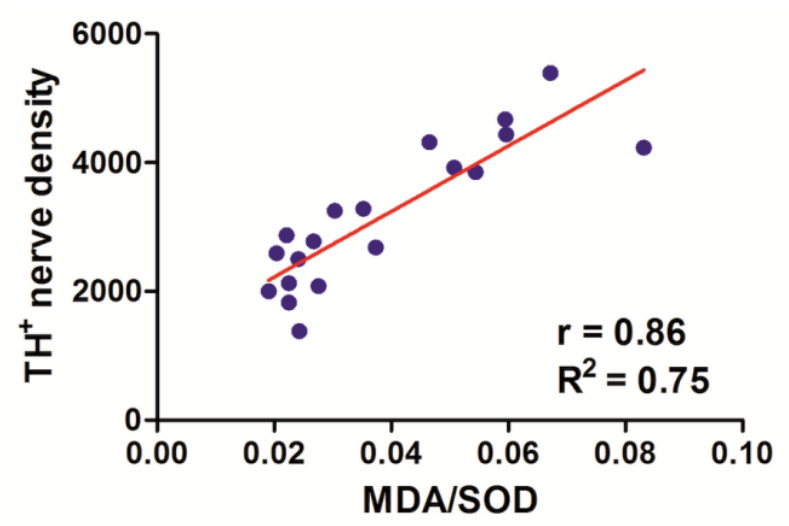

Figure 3. Correlation of the density of TH-positive nerve with the MDA/SOD ratio in control group and CSCs group $(n=19)$.

\subsection{Effect of Cardiac Stem Cells Transplantation on NGF Expression}

NGF expression was significantly higher in the control group $(1.10 \pm 0.15)$ compared with the sham group $(0.40 \pm 0.07, P<0.01)$. CSCs treatment resulted in lower NGF expression in the peri-infarct zone compared with the control group ( $0.84 \pm 0.13, P<0.05$ vs. control group. Fig. 4$)$.
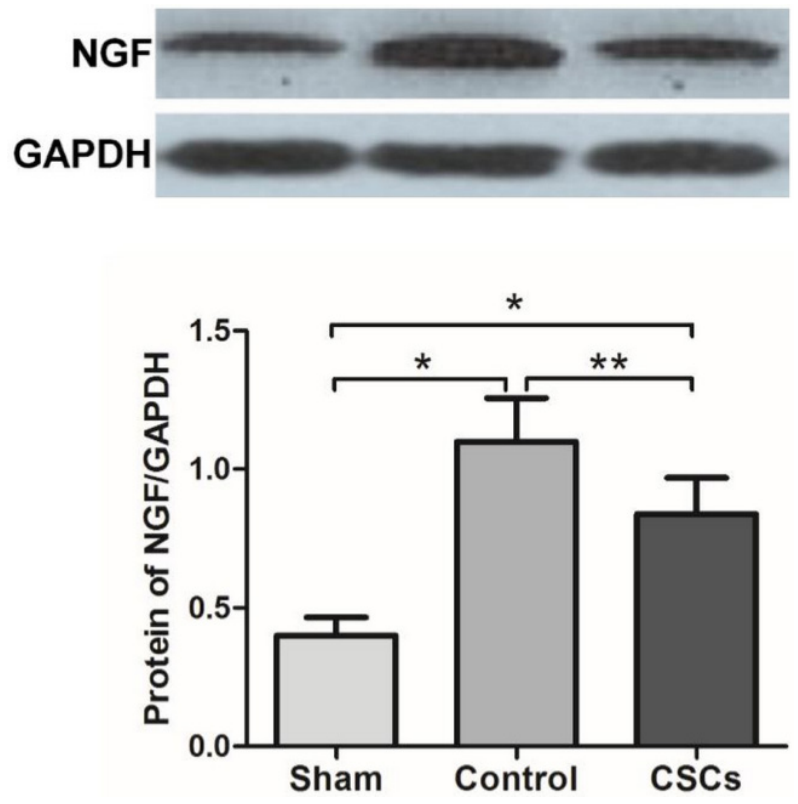

Figure 4. Representative western blotting bar for NGF 4 weeks after intramyocardial injection, with bar graph showing mean level of each protein expression. All data are expressed as mean $\pm \mathrm{SD}, *$ denotes $P<0.01$, ** denotes $P<0.05$.

\section{Discussion}

This is the first study investigate the protective effects of the CSCs against sympathetic remodeling after MI. Our data indicates that CSCs transplantation at peri-infarct zone significantly suppressed sympathetic remodeling via attenuated oxidative stress and NGF expression.

\subsection{Decreased NGF Expression Contributes to Suppressed Neural Remodeling}

Sympathetic remodeling, characterized by heterogeneous cardiac nerve sprouting and sympathetic hyper innervation, is a known substrate for lethal ventricular arrhythmias and SCD [18-20]. Sympathetic nerve activation exerts significant effects on electrophysiological properties such as triggered activity, automaticity, refractoriness, and conduction velocity of cardiomyocytes [21]. Although it is still unclarified that the precise mechanism of sympathetic remodeling after MI, it is generally agreed that NGF is strongly involved in this pathological process. MI results in immediate local NGF release, followed by upregulation of NGF expression at the peri-infarct zone [20] which persists for 2 months post MI in mice [22]. NGF infusion to the left stellate ganglion in dogs with chronic MI and AV block enhances sympathetic remodeling and induces spontaneous ventricular arrhythmias and SCD [19]. Furthermore, inhibition of proinflammatory response and NGF signaling suppresses neural remodeling and sympathetic hyperinnervation [2]. Consistent with previous studies [22, 23], we also detected NGF protein expression was significantly increased post MI. The NGF expression was markedly decreased in CSCs treated group, which could contribute to the reduction of sympathetic remodeling.

\subsection{Attenuated Oxidative Stress Results in Decreased Nerve Growth Factor Expression}

Increasing evidences indicate enhanced oxidative damage and inflammation result in sympathetic remodeling by upregulation of NGF expression [5, 24]. It is reported inflammatory cells and cardiomyocytes around the myocardial injury after MI are the important sources for NGF synthesis [5, 14, 24, 25]. In our previous study [14], paracrine factors secreted by mesenchymal stem cells (MSCs) lead to increased NGF expression in cardiomyocytes. Furthermore, NGF expression regulated by endothelin-1 in cardiomyocytes was observed [25]. On the other hand, inflammatory cells are the other major source for generating NGF. The increased expression of NGF was mainly localized within peri-infarct zone where accumulation of macrophages and myofibroblasts were present [24]. The destruction of macrophage, in the study [5], markedly reduced post-infarction levels of NGF following MI. Our data indicated that $\mathrm{MI}$ increased $\mathrm{MDA} / \mathrm{SOD}$ ratio which is an index of oxidative stress, and CSCs transplantation decreased oxidative stress level. There was a consistent 
pattern of NGF expression indicating that the close relationship between oxidative stress and NGF expression. Furthermore, in our study, the level of oxidative stress was positively correlated with sympathetic remodeling probably via the level of NGF in peri-infarct zone.

Our data also indicated oxidative stress was significantly activated by MI induction, but which was suppressed by CSCs transplantation. This is consistent with previous studies, in which the anti-oxidant effects of stem cells have been well reported. Yan [26] previously demonstrated that transplanted induced pluripotent stem cells attenuated oxidative stress and improve cardiac function through the Akt cell survival pathway in diabetic cardiomyopathy. Chen [12] showed adipose-derived MSCs suppressed oxidative stress and inflammatory reaction and protected kidneys against ischemia-reperfusion injury. Arslan [27] indicated MSCs-derived exosomes decrease oxidative stress and activate PI3K/Akt pathway to enhance myocardial viability and prevent adverse remodeling after myocardial ischemia/reperfusion injury.

Taken together, we demonstrated CSCs suppressed cardiac nerve sprouting in a rat model of MI. This implied one of the underlying mechanisms of improvement of cardiac electrophysiological stability post MI treated with CSCs described in our previous study [11]. However, our other previous study [14] showed MSCs enhanced cardiac nerve sprouting via NGF expression promoted by paracrine factors of MSCs. We postulated there is a balance between suppression of oxidative stress which downregulated NGF expression and paracrine factors which increased NGF expression [25]. As for CSCs, it may have stronger ability to suppress oxidative stress and less special paracrine factors. This may be one of the intrinsic differences between CSCs and MSCs.

\section{Conclusions}

Our study demonstrated that the protective effect of CSCs against sympathetic remodeling induced by MI was probably related to the attenuated oxidative stress accompanied by inhibition of NGF expression in rats. Thus, our study implied the underlying mechanism of improvement of cardiac electrophysiological stability post MI treated with CSCs.

\section{Acknowledgements}

The authors do not report any potential conflict of interest relevant to this article. This study was supported by grants from the Natural Science Foundation of Guangdong Province (S2013040014921) and the Science and Technology Planning Project of Guangdong Province, China (2014A020212088).

\section{Competing Interests}

The authors declare they have no competing interests.

\section{REFERENCES}

[1] Korngold EC, Januzzi JL, Jr., Gantzer ML, Moorthy MV, Cook NR, Albert CM. Amino-terminal pro-B-type natriuretic peptide and high-sensitivity C-reactive protein as predictors of sudden cardiac death among women. Circulation. Jun 9 2009; 119(22):2868-2876.

[2] Yuan MJ, Huang CX, Tang YH, et al. A novel peptide ghrelin inhibits neural remodeling after myocardial infarction in rats. Eur J Pharmacol. Sep 15 2009; 618(1-3):52-57.

[3] Shen MJ, Zipes DP. Role of the autonomic nervous system in modulating cardiac arrhythmias. Circ Res. Mar 14 2014; 114(6):1004-1021

[4] Fukuda K, Kanazawa H, Aizawa Y, Ardell JL, Shivkumar K. Cardiac innervation and sudden cardiac death. Circ Res. Jun 5 2015; 116(12):2005-2019.

[5] Wernli G, Hasan W, Bhattacherjee A, van Rooijen N, Smith PG. Macrophage depletion suppresses sympathetic hyperinnervation following myocardial infarction. Basic Res. Cardiol. Nov 2009; 104(6):681-693.

[6] Kimura K, Ieda M, Fukuda K. Development, maturation, and transdifferentiation of cardiac sympathetic nerves. Circ Res. Jan 20 2012; 110(2):325-336.

[7] Huang B, Scherlag BJ, Yu L, Lu Z, He B, Jiang H. Renal sympathetic denervation for treatment of ventricular arrhythmias: a review on current experimental and clinical findings. Clin Res Cardiol. Jan 182015.

[8] Liu YB, Wu CC, Lu LS, et al. Sympathetic nerve sprouting, electrical remodeling, and increased vulnerability to ventricular fibrillation in hypercholesterolemic rabbits. Circ Res. May 30 2003; 92(10):1145-1152.

[9] Leri A, Kajstura J, Anversa P. Cardiac stem cells and mechanisms of myocardial regeneration. Physiological reviews. 2005; 85(4):1373.

[10] Quijada P, Salunga HT, Hariharan N, et al. Cardiac Stem Cell Hybrids Enhance Myocardial Repair. Circ Res. Sep 25 2015; 117(8):695-706.

[11] Zheng S, Zhou C, Weng Y, et al. Improvements of cardiac electrophysiologic stability and ventricular fibrillation threshold in rats with myocardial infarction treated with cardiac stem cells. Crit. Care Med. May 2011; 39(5):1082-1088.

[12] Chen YT, Sun CK, Lin YC, et al. Adipose-derived mesenchymal stem cell protects kidneys against ischemia-reperfusion injury through suppressing oxidative stress and inflammatory reaction. J Transl Med. 2011; 9:51.

[13] Smith RR, Barile L, Cho HC, et al. Regenerative potential of cardiosphere-derived cells expanded from percutaneous endomyocardial biopsy specimens. Circulation. Feb 20 2007; 115(7):896-908.

[14] Chen J, Zheng S, Huang H, et al. Mesenchymal Stem Cells Enhanced Cardiac Nerve Sprouting via Nerve Growth Factor in a Rat Model of Myocardial Infarction. Curr Pharm Des. 2014; 20(12):2023-2029. 
[15] Li W, Ma N, Ong LL, et al. Bcl-2 engineered MSCs inhibited apoptosis and improved heart function. Stem Cells. Aug 2007;25(8):2118-2127.

[16] Zhou SX, Zhou Y, Zhang YL, Lei J, Wang JF. Antioxidant probucol attenuates myocardial oxidative stress and collagen expressions in post-myocardial infarction rats. J Cardiovasc Pharmacol. Aug 2009; 54(2):154-162.

[17] Sun SJ, Wu XP, Song HL, Li GQ. Baicalin ameliorates isoproterenol-induced acute myocardial infarction through iNOS, inflammation, oxidative stress and P38MAPK pathway in rat. International journal of clinical and experimental medicine. 2015; 8(12):22063-22072.

[18] Cao JM, Fishbein MC, Han JB, et al. Relationship between regional cardiac hyperinnervation and ventricular arrhythmia. Circulation. Apr 25 2000; 101(16):1960-1969.

[19] Cao JM, Chen LS, KenKnight BH, et al. Nerve sprouting and sudden cardiac death. Circulation Research. Apr 14 2000; 86(7):816-821.

[20] Zhou S, Chen LS, Miyauchi Y, et al. Mechanisms of cardiac nerve sprouting after myocardial infarction in dogs. Circulation Research. Jul 9 2004; 95(1):76-83.

[21] Opthof T, Misier AR, Coronel R, et al. Dispersion of refractoriness in canine ventricular myocardium. Effects of sympathetic stimulation. Circ Res. May 1991;68(5):1204-1215.
[22] Oh YS, Jong AY, Kim DT, et al. Spatial distribution of nerve sprouting after myocardial infarction in mice. Heart Rhythm. Jun 2006;3(6):728-736.

[23] Xin P, Pan Y, Zhu W, Huang S, Wei M, Chen C. Favorable effects of resveratrol on sympathetic neural remodeling in rats following myocardial infarction. Eur J Pharmacol. Sep 24 2010.

[24] Hasan W, Jama A, Donohue T, et al. Sympathetic hyperinnervation and inflammatory cell NGF synthesis following myocardial infarction in rats. Brain Res. Dec 8 2006; 1124(1):142-154.

[25] Ieda M, Fukuda K, Hisaka Y, et al. Endothelin-1 regulates cardiac sympathetic innervation in the rodent heart by controlling nerve growth factor expression. The Journal of Clinical Investigation. Mar 2004; 113(6):876-884.

[26] Yan B, Singla DK. Transplanted induced pluripotent stem cells mitigate oxidative stress and improve cardiac function through the Akt cell survival pathway in diabetic cardiomyopathy. Mol Pharm. Sep 3 2013; 10(9):3425-3432.

[27] Arslan F, Lai RC, Smeets MB, et al. Mesenchymal stem cell-derived exosomes increase ATP levels, decrease oxidative stress and activate PI3K/Akt pathway to enhance myocardial viability and prevent adverse remodeling after myocardial ischemia/reperfusion injury. Stem Cell Res. May 2013;10(3):301-31 